\title{
Two-arm haptic force-feedbacked aid for the shoulder and elbow telerehabilitation
}

\author{
Patrick Salamin ${ }^{1}$, Daniel Thalmann ${ }^{1}$, Frédéric Vexo $^{1}$, and Stéphanie Giroud ${ }^{2}$ \\ 1 VRLab - EPFL, Switzerland, \\ WWW home page: http://vrlab.epfl.ch \\ 2 Hôpital du Chablais, \\ WWW home page: http://www.hopitalduchablais.ch
}

\begin{abstract}
In this paper we present a telerehabilitation system aiming to help physiotherapists on the shoulder and elbow treatment. Our system is based on a two-arm haptic force feedback to avoid excessive efforts and discomfort with the spinal column and is remotely controlled by smart phone. The validation of our system, with the help of muscular effort measurements (EMG) and supervised by a physiotherapist, provides very promising results.
\end{abstract}

\section{Introduction}

Patients suffering from muscle or ligament diseases need training for their rehabilitation after the medical diagnostic. This kind of treatment can be applied only in a hospital or in a physiotherapist office. As mentioned in [2], having a machine able to handle the patients in (even rural) hospitals would then be very useful, but there is still a need of a physiotherapist during the treatment. If the patients want to get the best rehabilitation, they need an expert physiotherapist for their injured limb. Unfortunately, these physiotherapists are most of the times working in the metropolis because of their popularity. It would thus be a strong asset if they could remotely control these machine from their office. With such a system, the physiotherapists would easily train to better rehabilitate the patients almost at home.

In this paper, we propose a remotely controlled system for patient shoulders and elbows rehabilitation and training of physiotherapists. We decide to work with the Immersion ${ }^{3}$ Haptic Workstation ${ }^{\mathrm{TM}}$ instead of other VR haptic devices. In fact, it is a two-armed-based system [11 that allows a well-balanced effort of the patients between their right and left limbs. This avoids excessive torsions and efforts with the spinal column. Moreover, this machine can be easily tele-operated by a smartphone via Internet in contrary of other "home medical systems" like the Biodex ${ }^{4}$ or the Cybex ${ }^{5}$ that are not less cumbersome.

3 http://www. sensable.com

4 http://www.biodex.com/rehab/rehab.htm

5 http://ecybex.com 
We first present a short overview of the related fields that lead us to this improved system. Secondly, we describe the overall system and its implementation. The third part is dedicated to the system validation by a physiotherapist with the help of an ElectroMyoGraph (EMG) to measure the muscular effort of the patient. Finally, we conclude with the advantages of our system and its future possible improvements.

\section{Related works}

Using machines as an aid for the rehabilitation is not a new concept. Researchers already thought about it in 1965 [13] but only the veterans were targeted till 1967 [7]. In the following years, researches tends to aid handicapped people to substitute their "damaged" limbs [4.

Since the beginning of this millennium, rehabilitation became a very fashion topic. An overview of the current machines aiding to rehabilitation can be found in [16. Researchers now also work on solutions for incapacitated people in order to help them to recover the use of their limbs that we split into two parts: the lower upper limbs. These last ones can be divided in four main parts that have to be considered: shoulder, elbow, wrist and fingers. Tsagarakis et al. 114 developed one of the first machines aiding to people rehabilitation. Unfortunately, the applied forces were bounded to $2 \mathrm{kgs}$, which limited a lot the efficiency of the prototype for a complete treatment.

David Jack et al. focused on the fingers rehabilitation with the help of forcefeedback gloves 6. Others researchers made complementary works and developed a $6 \mathrm{DOF}$ machine for the shoulder, elbow and wrist rehabilitation for one hand at a time 3 . Nevertheless, all these devices require the presence of a doctor during the patient healing. In 2005, Demiris et al. highlighted in [2] the interest to develop a machine that would allow healing the patient at distance. They involved by this way the telerehabilitation in the scientific research for the patients' well-being.

Based on these researches and with the help of a physiotherapist, we assumed that the HW would be very efficient and promising to treat upper limbs injuries like the shoulders and the elbows. We present our system in the following chapter.

\section{System description and implementation}

We present hereinafter our application and the artifacts that should improve its believability and efficiency.

\subsection{System architecture}

As the goal of this application is the telerehabilitation, we want to allow the physiotherapists to be completely mobile and independent from the patient location. A PDA with an integrated webcam (e.g. a PDA-phone) seems thus to perfectly 
fit to our requirements. By this way, the patient can see a video-streaming of the physiotherapist sent by the PDA during its treatment. The physiotherapist is informed of the patient arms location with a 3D interface created through the MVisio 3D graphics engine 12 and the data sent by the HW. As you can see on the first picture from the left on the Figure 1 the physiotherapist does not need to be with the patient or in his/her office to begin the treatment.

The patient is wearing a HMD while being seated in the HW as shown on the third picture from the left on the Figure 1. The HW allows the therapist to apply different forces in a range of 0 gram to $10 \mathrm{k}$ grams in every direction on each arm independently. As we can see in 15, the shoulder can be injured in several ways. It is thus useful to be able to apply accurate forces in order to help him/her to move his/her arm at the beginning and then to apply a force against his/her movement. As the HW applies the forces on the wrist and as we want the user to move his/her shoulder, we hold the patient arm straight with the help of a harness. The main advantage of using the HW - even if it is very expensive - resides in the possibility to apply forces on both arms. By this way, if we apply symmetrical forces on both arms, the patient will not try to twist his/her trunk to execute the movement. The rehabilitation would then not lead to new problems with the spinal column.

Moreover, as we are working with the HW, with the help of the paradigm developed by Renaud Ott in [10, it is possible to compensate the gravity effect as if the patient arm was in weightlessness. The patients can be treated from the beginning with micro-gravity to their complete recovery with forces around $10 \mathrm{kgs}$. It is also very interesting for the physiotherapist to be able to apply a constant and exact force (less than one gram). This would avoid most of errors due to human factor (deviance of the applied force that is neither exact nor constant).

Finally, electrodes are applied on the patient skin to detect the muscles activity with the help of an EMG. The provided information is sent to the physiotherapist who can better evaluate and appreciate the force to apply on the patient and the evolution of his/her rehabilitation.
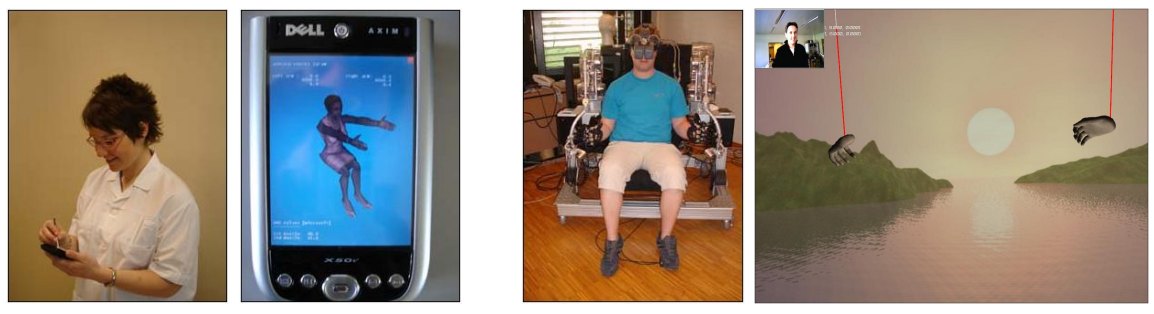

Fig. 1. On the left, the physiotherapist (hardware and interface); on the right, the patient (hardware and interface) during the rehabilitation exercises 


\subsection{Improvements for the user immersion}

The physiotherapist only needs to have a webcam and a PDA with a very simple interface as you can see on the second picture from the left on the Figure 1. $\mathrm{He} /$ she can see the current patient position represented by an avatar. The doctor can also easily change the forces applied on the patient with the help of the stylus by indicating the concerned wrist and the force direction and amplitude which depends on the line he/she draws (values in grams written on the screen).

On the patient side of our application, we know by experience that VR systems are quite invasive and can stress the user. The simulation would then be less efficient, traumatizing and even harmful for the patient. This is why the user is immerged in a virtual environment with a relaxing landscape like a beach or mountains, depending on his/her preference as you can see on the right picture of the Figure 1. A sweet relaxing music as background noise also contribute to strongly reduce the patient anxiety due to the VR machines [8].

Moreover, the patient can see a realistic representation of his/her hands which follows in real-time the real position and orientation of the real ones. Notice that the force is applied on the user wrist(s) is represented as for puppets: the concerned wrist is caught by a wire. With this artifact, the patient can see (right picture of the Figure 1 in which direction the force is applied and also its intensity (a second wider red wire indicates it). All these artifacts improve the immersion of the patient who could be seriously perturbed by the used hardware that we present in the following section.

Once done, some scenarios could be easily added to the simulation. For example, the user could have to touch a virtual ball the physiotherapist would move around specific places. It has already been proved that such a playful simulation improves rehabilitation results [1] 9].

Finally, during the simulation, the patient has a "Window to the World" which allows him/her to see the physiotherapist and to listen to him/her during the session. As shown in [5], it is very relaxing for the patient to have a multimodal link with the doctor while being in the virtual environment.

In order to prove the efficiency of our system we made some tests that are presented in the following section.

\section{Experiments}

We display a relaxing landscape during the complete session. Moreover, we first let the patient in the environment with a sweet music in background during fifteen minutes in order to compensate the stress possibly brought by the VR engines. Once the user seems to be relaxed, we begin the treatment. We decide to call "neutral position" the position in which a tester tighten ones' arms close to one's trunk on the vertical plane without moving.

Obviously, depending on his/her recovery status, a help (or a constraint) is applied by the HW when the patient tries to perform the asked movements shown on the Figure 2. We have to remind that for every exercise, the patient 
moves both arms in a synchronous and parallel way (or symmetrical when they are on the same plane, e.g. for the abduction we present hereinafter) to avoid useless and dangerous efforts and torsions on the spinal column.
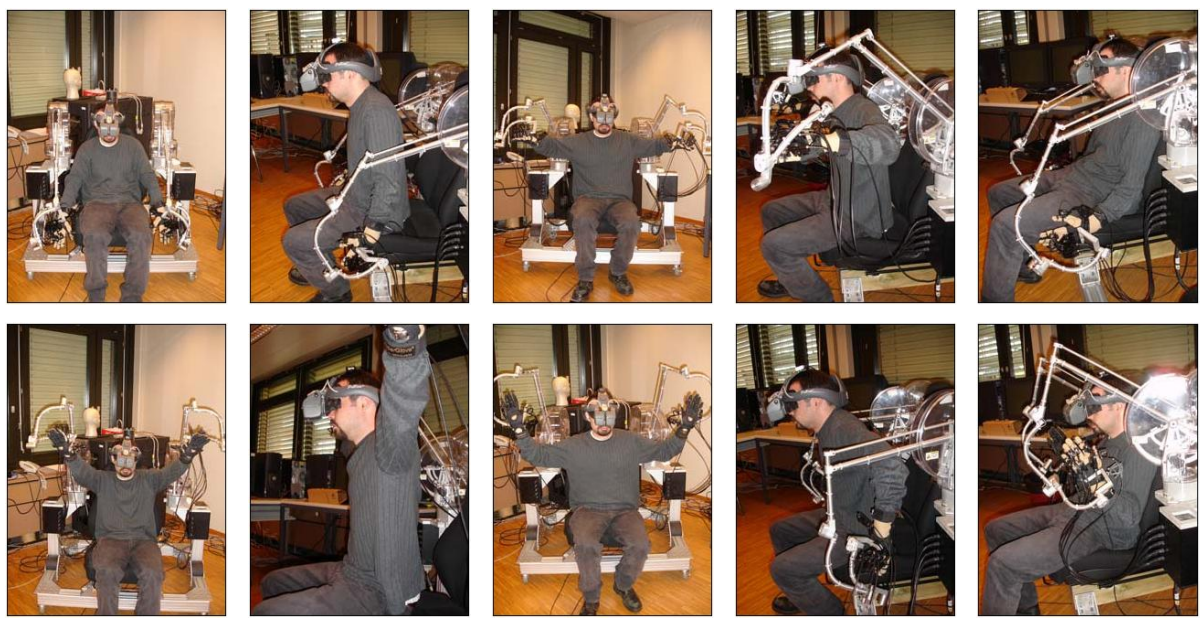

Fig. 2. Exercises for the rehabilitation (from the left to the right): inflection, abduction, lateral rotation, medial rotation for the shoulder and elbow inflection

We present hereinafter four exercises for the shoulder rehabilitation: inflection, abduction, lateral and medial rotation. Eight testers (two females) participate to the simulation to check the efficiency and the limitations of our system, but unfortunately (or hopefully) no one had any shoulder or elbow troubles.

The inflection exercise consists in moving ones arm in a vertical plane ahead of oneself as shown on the left pictures of the Figure 2 The concerned muscles are mainly the anterior deltoid and the pectoralis major, but the coraco brachial and biceps are also working during this exercise. In the following section, you will see that we put electrodes on the main-working muscles to check their activity during the tests.

In the abduction exercise, as you can see on the second pictures from the left of the Figure 2, the patient must move his/her arms in a vertical plane on his/her sides (instead of ahead). In this experiment, the most acting muscles are the middle deltoid and the supraspinatus, whose behavior will be observed and commented in the following section.

Concerning the rotation exercises, the patient arms start in position shown on the top third and fourth pictures from the left in the Figure 2 For the lateral rotation, the forearms raise up to the vertical. In this case, the main working muscles are the posterior deltoid, the teres mino and infraspinatus which are working together (bottom centered picture). For the medial rotation, the forearms fall down (almost) to the vertical. The main detectable activated muscles 
are the anterior deltoid and the pectoralis major but some others like the subscapularis (under the pectoralis major), the latissimus dorsi and the teres major are also working but less perceptible with the EMG.

Concerning the elbow rehabilitation (right pictures in the Figure 2, we first assume the patient keeps the arm in the "neutral position" (close to the body trunk). After this, he/she must move vertically in a synchronous way the forearms from the bottom to the top and vice versa. The most important muscles used to perform this exercise are the biceps and the triceps on which we also put electrodes in order to obtain their activity. We present in the next section typical results we obtained during our sessions with the help of our system and an EMG.

\section{Discussion of results}

As written before, in order to avoid bias due to the VR devices used for these experiments, the user is first seating in the $\mathrm{HW}$ with sweet music and a relaxing landscape during fifteen minutes. He/she performs then twice the exercise, e.g. "down - up - down - up - down" for the shoulder inflection. We present hereinafter the results we obtained but, in order to better understand the provided graphs, we first define two terms concerning the muscles:

- effectors: These muscles are used to execute a movement. They give the possibility to the patient to accomplish a movement. If, in our case, the shoulder does not move, they do not act. Their EMG representation has a big difference when the shoulder moves or not.

- stabilizers: These muscles are always in activity, even when the shoulder does not move because they have to stabilize the joint to avoid (e.g. a dislocation due to the gravity effect). They almost do not act to perform a movement. Their EMG representation seems to be almost constant.

For the first exercise we present, the inflection, the electrodes are located on the anterior deltoid and the pectoralis major. They are respectively represented on the EMG graphs by the red and the blue lines on the Figure 3. Notice that we can perfectly see the role of those muscles: both are effector muscles and work during the whole movement (up - stay - down) while the patient arm is not in the "neutral position". We can also notice that the main used muscle for the inflection is the anterior deltoid while the pectoralis major only helps it for this action. It is also proved on the right graph of the right picture of the Figure 3 because when a force is applied against the patient movement, the pectoralis major acts during the complete movement.

The abduction exercise mainly involves the activity of the middle deltoid (in red) and the supraspinatus (in blue). Their activity during the experiment can be seen on the Figure 4. Once again, we decided to check the activity of an effector (the deltoid) and a stabilizer (the supraspinatus) to verify if the $\mathrm{HW}$ is really efficient when it helps or counter to the patient movements. We can see on the Figure 4 that the muscles, during the movement, are used a lot - even with 

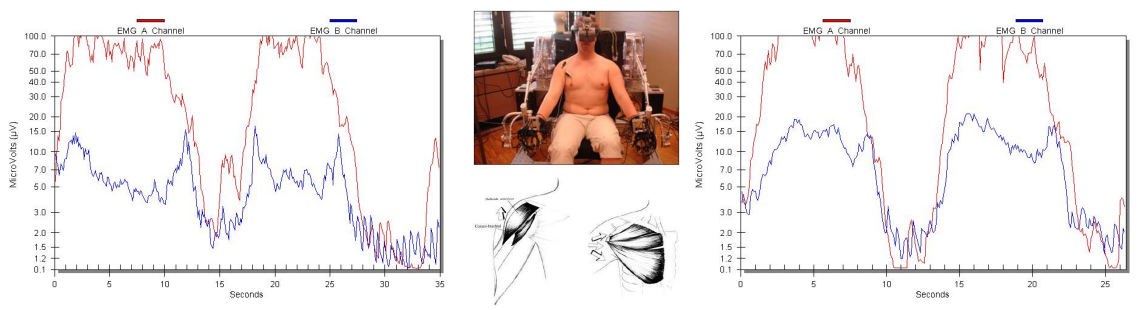

Fig. 3. Inflection exercise for the shoulder: (left)with help, (right)countered to and (center) position of the electrodes (anterior deltoid on the bottom left and pectoralis major on the right)

the help provided by the HW. But we can also remark that they are almost not used when the user is in the "neutral position".
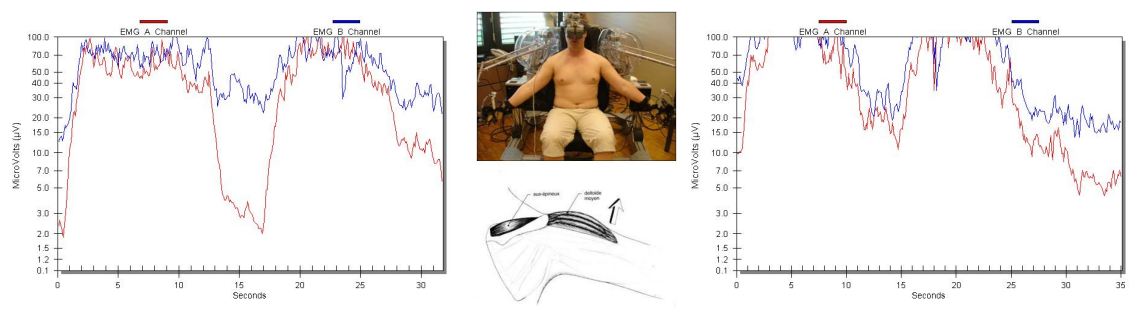

Fig. 4. Abduction exercise for the shoulder: (left) with help, (right) countered to and (center) position of the electrodes (supraspinatus on the bottom left and middle deltoid on the right)

For the lateral rotation (Figure 5), the electrodes are located on the posterior deltoid (blue line) and both teres minor and infraspinatus because they are linked(red line). As those last ones are stabilizer muscles, they seem to be always active during the experiment while the posterior deltoid can "have a rest" when the patient is in the "neutral position". In this case, the main difference of muscle activity appears for the stabilizer ones because the applied countering forces are strong (right graph of the Figure 5).

The medial rotation (Figure 6) involves lots of muscles. The anterior deltoid the pectoralis major are the most active and interesting to analyze with an EMG. Among the others, we can cite the subscapularis, the altissimus dorsi and the teres major. As we can see it on the left and right pictures, both analyzed muscles are effector ones (only the subscapuralis is a stabilizer). They thus almost follow the same curve and we can see that the forces applied by the HW are also quite efficient for this exercise. Notice that for this exercise, the applied counter forces are pointing ahead and up. 

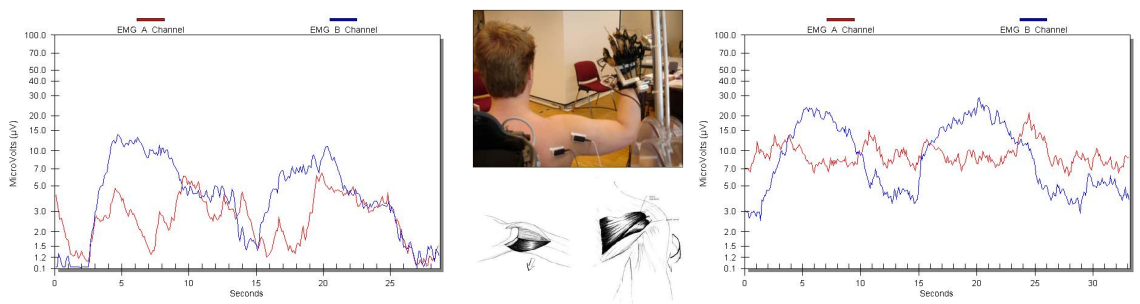

Fig. 5. Lateral rotation exercise for the shoulder: (left) with help, (right) countered to and (center) position of the electrodes (posterior deltoid on the bottom left, teres minor and infraspinatus on the right)
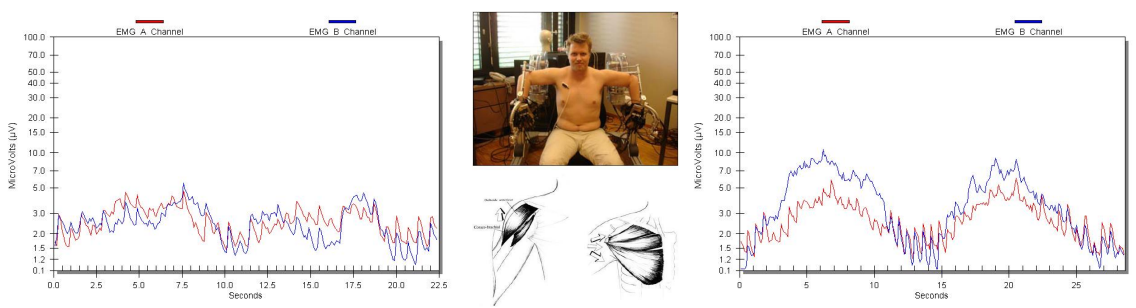

Fig. 6. Medial rotation exercise for the shoulder: (left) with help, (right) countered to and (center) position of the electrodes (anterior deltoid on the bottom left, pectoralis major and subscapularis (hidden) on the right)

The last exercise we present in this paper concerns the elbow rehabilitation (Figure 7). We ask the patient to perform elbow inflections and we measure the biceps (red line) and triceps (blue line) activity. It is interesting to see that the triceps, which is normally an effector muscle for the extension of the elbow, also acts in this exercise and almost follow the biceps curve. Otherwise, the efficiency of the HW and the applied force is obvious. A big difference between the muscle activity between the left (helping forces) and the right graphs where a force is applied to the front and to the bottom can be noticed.

Finally, we prove the benefits of working with both arms at the same time. We have made this assumption because when a physiotherapist asks a patient to do rehabilitation exercises at home, he/she often propose to perform them with both hands. We then check the activity of the errector spinea (shown in the center of the Figure 8) for the abduction exercise we have already presented. We locate an electrode on the left side (red line of the graph below) of the spinal column and another one on the right side (blue line). In order to obtain the graph on the Figure 8, we first asked the patient to stick up his/her arm and then to lower it. After this, he/she performs the same movement with the other arm and finally with both together. As you can see below, there is a very big difference between the right and left spinal muscles activity when the patient only sticks up 

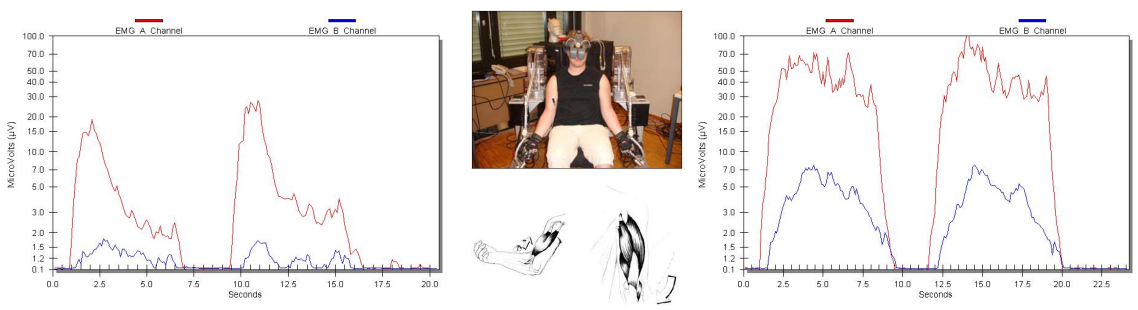

Fig. 7. Inflection exercise for the elbow: (left) with help, (right) countered to and (center) position of the electrodes (biceps on the bottom left and triceps on the right)

one arm. This leads to spinal column torsion and often to backache. This graph prove then the importance to work with both arms during the rehabilitation.
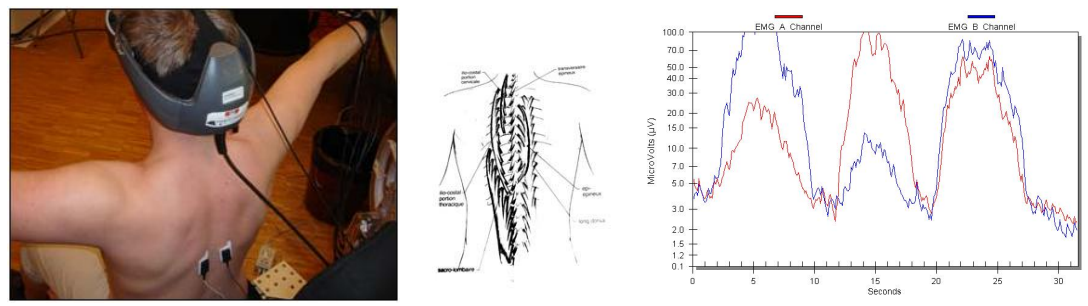

Fig. 8. Back muscles difference

\section{Conclusion}

The goal in this paper was to present an efficient aid for the shoulder and elbow telerehabilitation. Our application full-fills the tele-operation side that provides obvious advantages for the patients and the physiotherapists training. Sometimes an adaptation time of five to ten minutes was needed to discover the VR material, but none of the patients were really perturbed by them during the simulation. Furthermore, the obtained results seem to prove the efficiency of our system for the patients during all the rehabilitation phase. We can e.g. see in the graphs that a very light force is needed to perform the action when the patient starts his/her rehabilitation. And when he/she has almost recovered all his/her faculties, the HW can apply strong enough forces on his/her arms to finish correctly the rehabilitation. The EMG last graphs also support the idea of the minimal spinal column torsions mandatory for the patient comfort. Moreover, the possibility to cure the patients at distance also really interested our physiotherapist. This technology extends the coverage of this kind of therapy because the patients can be treated in any hospital even if the therapist is not physically present. 
Concerning the future works, it could be really interesting, e.g. for the lateral and medial rotation, to provide a support for the elbow during the therapy. The physiotherapist should also take into account an additional five to ten minutes to install the patient in the HW. However this task can be performed by any member of the medical staff.

\section{Acknowledgments}

This work has been partially founded by the EU IST-INTUITION Network Of Excellence.

\section{References}

1. A. L. Betker, T. Szturm, Z. K. Moussavi, and C. Nett. Game based interactive motivating tool for rehabilitation movements: case study. In ACST'06: Proceedings of the 2nd IASTED international conference on Advances in computer science and technology, pages 157-162, Anaheim, CA, USA, 2006. ACTA Press.

2. G. Demiris, C. L. Shigaki, and L. H. Schopp. An evaluation framework for a rural home-based telerehabilitation network. J. Med. Syst., 29(6):595-603, 2005.

3. J. Furusho, C. Li, X. Hu, N. Shichi, T. Kikuchi, A. Inoue, K. Nakayama, Y. Yamaguchi, and U. Ryu. Development of a 6-dof force display system using er actuators with high-safety. In VRCIA Ó6: Proceedings of the 2006 ACM international conference on Virtual reality continuum and its applications, pages 405-408, New York, NY, USA, 2006. ACM.

4. W. J. Greenleaf. Applying vr to physical medicine and rehabilitation. Commun. ACM, 40(8):42-46, 1997.

5. M. Gutiérrez, P. Lemoine, D. Thalmann, and F. Vexo. Telerehabilitation: controlling haptic virtual environments through handheld interfaces. In VRST '04: Proceedings of the ACM symposium on Virtual reality software and technology, pages 195-200, New York, NY, USA, 2004. ACM.

6. D. Jack, R. Boian, A. Merians, S. V. Adamovich, M. Tremaine, M. Recce, G. C. Burdea, and H. Poizner. A virtual reality-based exercise program for stroke rehabilitation. In Assets '00: Proceedings of the fourth international ACM conference on Assistive technologies, pages 56-63, New York, NY, USA, 2000. ACM.

7. D. L. Jaffe. An overview of programs and projects at the rehabilitation research and development center. In Assets '94: Proceedings of the first annual ACM conference on Assistive technologies, pages 69-76, New York, NY, USA, 1994. ACM.

8. K. Kallinen. The effects of background music on using a pocket computer in a cafeteria: immersion, emotional responses, and social richness of medium. In $\mathrm{CHI}$ '04: CHI '04 extended abstracts on Human factors in computing systems, pages 1227-1230, New York, NY, USA, 2004. ACM.

9. C. Lathan, J. M. Vice, M. Tracey, C. Plaisant, A. Druin, K. Edward, and J. Montemayor. Therapeutic play with a storytelling robot. In CHI '01: CHI '01 extended abstracts on Human factors in computing systems, pages 27-28, New York, NY, USA, 2001. ACM.

10. R. Ott, M. Gutierrez, D. Thalmann, and F. Vexo. Improving user comfort in haptic virtual environments through gravity compensation. In WHC '05: Proceedings of the First Joint Eurohaptics Conference and Symposium on Haptic Interfaces for 
Virtual Environment and Teleoperator Systems, pages 401-409, Washington, DC, USA, 2005. IEEE Computer Society.

11. R. Ott, V. D. Perrot, D. Thalmann, and F. Vexo. Mhaptic : a haptic manipulation library for generic virtual environments. $c w, 0: 338-345,2007$.

12. A. Peternier, D. Thalmann, and F. Vexo. Mental vision: a computer graphics teaching platform. In Edutainment 2006, Technologies for E-Learning and Digital Entertainment. First International Conference, Edutainment 2006. Proceedings (Lecture Notes in Computer Science Vol.3942), pages 223-232, 2006. Virtual Reality Lab., Ecole Polytech. Fed. de Lausanne, Switzerland.

13. G. Rose, M. A. Woodbury, and E. S. Fergusson. Recent study of teaching: machine aids in rehabilitation therapy. In Proceedings of the 1965 20th national conference, page 7, New York, NY, USA, 1965. ACM. Chairman-L. R. Turner.

14. N. G. Tsagarakis and D. G. Caldwell. Development and control of a "soft-actuated" exoskeleton for use in physiotherapy and training. Auton. Robots, 15(1):21-33, 2003.

15. D. H. K. Uhthoff. Shoulder injury and disability. Technical report, Orthopaedic Surgeon, 2002.

16. S.-C. Yeh, A. Rizzo, W. Zhu, J. Stewart, M. McLaughlin, I. Cohen, Y. Jung, and W. Peng. An integrated system: virtual reality, haptics and modern sensing technique (vhs) for post-stroke rehabilitation. In VRST '05: Proceedings of the ACM symposium on Virtual reality software and technology, pages 59-62, New York, NY, USA, 2005. ACM. 